\title{
Applications of artificial intelligence with cloud computing in promoting social distancing to combat COVID-19
}

\author{
Mohammed Ghadhban Al-Hamiri, Hayder Fadhil Abdulsada, Laith A. Abdul-Rahaim \\ Department of Electrical Engineering, University of Babylon, Hillah, Iraq
}

\begin{tabular}{l} 
Article Info \\
\hline Article history: \\
Received Apr 3, 2021 \\
Revised Oct 12, 2021 \\
Accepted Oct 18, 2021 \\
\hline
\end{tabular}

Keywords:

Artificial intelligence

Cloud computing

COVID-19

Social distancing

\begin{abstract}
The emergence of Coronavirus disease 2019 (COVID-19) disease and its rapid spread around the world has serious impacts on people's lives in addition to its effects on many aspects, including the economic and educational sectors. Researches have proved that social distance is effective in combating COVID-19. Maintaining social distance is hard to be handled by humans especially in crowded areas such as airports and campuses. So, there is a need to apply a robust and proactive design to manage this process automatically and smartly. This paper presents a design system to fight COVID-19 by maintaining the social distance with effective monitoring for suspected cases. This has been done using cloud computing and a framework including Arduino (node microcontroller unit (NodeMCU)) with several sensors. The operational aspects of this design system using cloud computing have been discussed. Generally, NodeMCU has been involved in checking the conditions, comparison processing, and communication with the webserver. Moreover, the webserver has been used for determining the maximum number of persons allowed to enter. The results state that this design system is effective in combating COVID-19 through maintaining the social distance and collecting information about suspected cases. This system is valuable, dependable, and stable since the whole process is contactless.
\end{abstract}

This is an open access article under the CC BY-SA license.

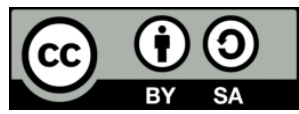

\section{Corresponding Author:}

Mohammed Ghadhban Al-Hamiri

Department of Electrical Engineering, University of Babylon

Hillah City, Babil, Iraq

Email: m.ghadhban@uobabylon.edu.iq

\section{INTRODUCTION}

The Coronavirus disease 2019 (COVID-19) has been spread over the world creating a serious challenge to humanity. Researchers are trying to contribute in combating this pandemic through new technologies such as cloud computing and artificial intelligence [1], [2]. Concepts, principles, and definitions do not change with time to some extent, unlike scientific research that is constantly developing. The definition of the term cloud computing varies for different researchers. Gartner defines cloud computing as a type of computing in which a large number of customers are given the ability to manage information technology (IT) applications with wide potential through an internet service [3]. The term cloud computing refers to any service provided to the cloud customer and it includes a wide range of useful applications that can be accessed anywhere and anytime on the basis of demand. The National Institute of Standards and Metrology Technology (NIST) defines cloud computing as an access model for computing resources that are available to share on-demand across the network such as servers, storage, software, and infrastructure that can be provided and released quickly with minimal administrative effort or in cooperation with a service provider [4], [5]. 
The technological development in the field of computing is not a result of chance or a sudden process, but rather it represents efforts and perseverance to meet the needs of the times. The operating system known as virtual machine (VM) was released in 1970 by International Business Machines Corporation (IBM), which was used by large and medium companies to operate several systems with memory and a special processing unit [6]. The invention of VM provided the appropriate environment for the development of many modern technologies such as virtualization and equipped a computing platform with different levels of cooperation such as parallel, central, cluster, and distributed computing, which resulted in cloud computing [7]. Cloud computing is presented in three dissimilar service models, each of which meets a specific set of customer needs [8]. These three models include:

- Software as a service (SaaS)

In this model, the operation, administration, and programming of applications and programs is the responsibility of the cloud computing service provider. The process of accessing the SAAS model by the clients is done through a web browser, which appears as an electronic application interface where the available services are used with the presence of the internet [9]. Convenient applications, for example, Gmail and Google Docs, can be accessed from the customer's computers, iPads, and mobile phones. The most important feature of SaaS is that the user does not have to purchase licenses or update or run all the programming on the computer. It also has different preferences, for example, increasing productivity through being able to lease it to multiple tenants, configurability, and adaptability [10]. Examples of SaaS vendors are Amazon web services, Google Apps, and Salesforce.com.

- Platform as a service (PaaS)

In this model, the provider delivers, manages, and maintains both framework and operating system programming. The client supervises the programming of applications and manages them in the framework and on the operating systems pre-programmed by the service provider. With this service model, the user does not have the authority to control the framework and the infrastructure [11]. As explained, SaaS gives the user ability to use applications without control or develop them. While PaaS enables the user to design, simulate, build and test online applications through the use of available resources such as programming languages, databases, and testing methods. PaaS provides a collaborative work environment between individuals or organizations from a distance. For example, PaaS enables users in different parts of the world to collaborate in building applications or websites. Notable PaaS providers are Windows Azure, Google Apps Engine, and Amazon Web Services [12].

- Infrastructure as a service (IaaS)

In this model, service providers equip computing resources for infrastructure engineers to install or run any program on these resources. Service providers are also responsible for creating application programming interfaces (APIs) that allow customers to access services from their devices [13]. With IaaS, computing, storage, and networking resources are consumed in a virtual way, meaning computing resources are transformed from a physical infrastructure to a virtual infrastructure. This virtual infrastructure is provided using virtualization technology. Service providers configure VMs that include virtual resources such as storage, network resources, and memory. Example of IaaS providers are Amazon Web Services (AWS), Google Compute Engine, and OpenStack [14].

Cloud computing became recently more related to another term which is the internet of things (IoT). The IoT can be defined as a network of household and physical devices as well as sensors, communication, and actuators that communicate collect and transfer data with each other [15]-[17]. The principle of work of the Internet of things depends on a large group of devices distributed in the environment that are connected either wired or wirelessly and that interact with each other to achieve the desired goals by providing new services and applications [18], [19]. The internet of things is enabled by artificial intelligence (AI), which is defined as a set of software algorithms that make devices process, make decisions, and perform human tasks [20], [21]. Generally, the aim of technology is to improve the life of humanity and reduce the risks that face the world such as COVID-19. The next sections state the real and likely employments of artificial intelligence in combatting COVID-19.

\section{LITERATURE REVIEW}

\subsection{Prediction and tracking}

AI has proven to be effective in following and predicting how the COVID-19 can spread over time and space [22]. The reports indicated that artificial intelligence had been used by a system (health map) at Boston Children's Hospital in the United States of America. This system issued its first warning on December 30, 2019 a half an hour before the warning from scientists at Program of Monitoring Emerging Diseases has been issued. Artificial intelligence has not yet been optimally utilized due to several reasons. The first reason is that the data on Coronavirus are still contradictory and inaccurate. For example, the availability 
of accurate data contributed to the prediction of the spread of Zika virus in 2015 using neural networks [23], [24]. The obstacles facing the exploitation of the big data collected from social media are considered a second reason for reducing the ability of artificial intelligence in predicting and tracking the spread of Corona disease.

\subsection{Control social distance}

The other role of artificial intelligence in fighting Coronavirus is to control social distancing. The measures of lockdown and social distancing have given health authorities permission to use artificial intelligence applications by using thermal imaging scans of individuals in public places [25]. Thus, it is possible to identify the infected persons whose temperature exceeds the normal limits and thus take preventive measures for infected people. With regard to individuals who violate the rules of social distancing, artificial intelligence can be used to recognize the faces and then send an alert for the purpose of urging them to obey the protection measures from the Coronavirus. Artificial intelligence technology can also be used to monitor the health status of patients and enable them to receive medical advice without the need to visit hospitals, thus contributing to social distancing [26].

The pandemic of COVID-19 is quickly increasing across the world. Each person can get infected in the future especially in poor contraries where there is no enough vaccine. Social distancing is going to turn into an effective way to avoid this pandemic by reducing the close physical contact among people which can minimize the probability of transferring the virus across the world. Technology has always made its positive contribution to issues of concern to humanity. For example, the use of robots dependent on the IoT to control social distancing and the use of deep learning techniques to identify signs of infection of the COVID-19. In addition, the blockchain application manages records of Coronavirus patients and mathematical models that contribute to predicting the spread of Coronavirus [27], [28].

Social distancing is the one of solution currently available in many countries of the world to prevent the rapid spread of the Coronavirus. Thus, the transmission of infection from an infected person to an uninfected person can be reduced by making people aware of the need to leave an appropriate distance between them. However, this process is not easy to apply on the ground due to the lack of sufficient spaces in public places or the required ventilation to purify the air. Some research suggests using a queue management application for people who are waiting for a specific service, as they are registered in the virtual waiting list without the need to collect customers in the actual waiting rooms with long queues for long periods. To conduct this process, machine learning is employed to estimate the time required for waiting, and thus customers are given the appropriate time to attend and receive the service. Also, previous data and artificial intelligence are used to arrange appointments so that customers can choose the right time to visit, taking into account preserving customers' privacy [29]. Actually, the correct prediction enables governments to take appropriate strategies and reduce possible sanitary measures [30].

Some research suggests using emerging technologies to maintain social distancing by diagnosing symptoms, monitoring and tracing infected people. Some of the techniques used to maintain social distancing are using the IoT, thermal scanning, and ultrasound. On the other hand, the policy of surveillance and tracking of persons has led to a set of challenges and legal problems in terms of preserving the privacy of individuals [31]. As a result of social distancing policies, this has led to the reliance on cloud computing for learning, work, and e-commerce purposes. The rapid and sudden transformation to the use of cloud computing has created challenges, especially for emerging and medium-sized companies. These challenges include how to maintain economic revenues, commercial growth, and enable employees to work remotely. In addition, the challenges of rapidly converting to the use of cloud computing without taking precautions and preventive measures lead to risks of an information breach, piracy, and cybersecurity [32].

At the present time, there are challenges with the spread of disease in crowded places such as an air station, railways, or universities, where the temperature test turns into a basic condition and it is not logically possible to generally rely on an individual to do the work. In addition, there is a high likelihood that an individual who checks a person's temperature to be affected by COVID-19 will become infected. Moreover, preventive measures limit the number of people present in one place, for example the classroom, where it becomes difficult to check the temperature of students and determine the number of entrants to the class in the traditional way, so it is necessary to use an innovative system to do this automatically. From previous researches, it is noticed that their proposed models and designs are reactive more than proactive. Based on the above problems, a novel technique is needed to deal with suspected cases in a way to preserve the social distance. On the other hand, this paper suggests a robustness design that is proactive through using advanced algorithms to deal with the surrounding environment. This paper presents a system design depending on cloud computing technology and using Arduino (node microcontroller unit (NodeMCU)) as a novel design to maintain social distance automatically and smartly. 


\section{RESEARCH METHOD}

The proposed system involves cloud computing which has been built using a web server (Apache) and database server (MySQL). This system is designed using hypertext preprocessor (PHP) code which works as an admin to manage the database. Apache, in turn, represents software that applies web server technique and its port is 80443 . Regarding structured query language (SQL), it represents the database server and its port is 3306. The cross-platform, Apache, MySQL, PHP and Perl (XAMPP) interface is involved which represents a package of servers. PHP, hypertext markup language (HTML), Javascript, and C++ codes are used in the proposed system to introduce a cloud interface. These codes also introduce links as application programming interfaces (API), so the devices (NodeMCU) that are involved in this system, can send data to these API links. Figure 1 shows the XAMPP interface which includes the used software, database server and their ports.

Regarding the hardware, two Arduinos (NodeMCU wireless fidelity (Wi-Fi)) have been used to build the system. The first one is called the main NodeMCU. This Arduino is attached with two ultrasonic sensors (one for entrance and one for exit), buzzer, solenoid lock, relay, and mlx90614 infrared radiation (IR) temperature sensor. The radio-frequency identification (RFID) is connected to another NodeMCU because all the pins of the first NodeMCU are used. On the other hand, the second NodeMCU is connected with the main NodeMCU by Wi-Fi through a router (TP-Link). Figure 2 displays the system hardware components which logically consist of external part and internal part. The external part includes the first ultrasonic sensor, IR temperature sensor, buzzer, and RFID reader, while the internal part includes the second ultrasonic, and solenoid lock.

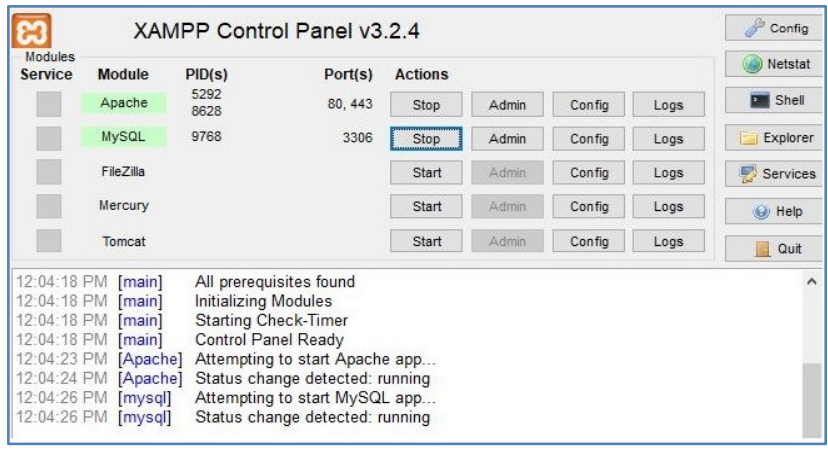

Figure 1. The XAMPP interface

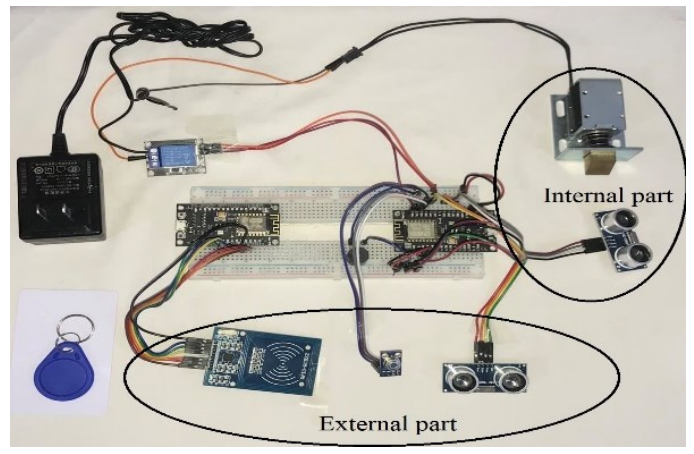

Figure 2. The system hardware components

As a first scenario in the web page, a push-button is placed for the system admin to open the door. When the admin pushes the button, NodeMCU will connect with a specific API link to check whether the door is open or not. Regarding the RFID sensor, when someone with RFID tag wants to enter, the secondary NodeMCU will send HTTP request to the web server. The web server, in turn, updates the table of variable in the database that the door is opened by making the variables equal to 1 . The main NodeMCU frequently checks with web server about the state of the door. If the state is one, the main NodeMCU will open the door through solenoid lock. Actually, this NodeMCU communicates with the database indirectly through the web server. This procedure is necessary to secure the database in the cloud. Web applications control the database in terms of adding, selecting, and updating. When the door is opened, NodeMCU sends HTTP request for the web server telling it to update the database and make the state of the door equal to zero.

As a second scenario, in case a person gets closer to the door where the outside ultrasonic is placed. There are conditions that must be satisfied to open the door in this case. The first condition is the number of students who existed in the classroom must be less or equal to the maximum number that is allowed. In this scenario, the maximum number of students who are allowed to exist at the same time is supposed to be 20 students. However, the maximum number can be controlled (increasing or decreasing) on the cloud page by the system admin. The main NodeMCU sends HTTP request to the web about the maximum number and the actual number of students inside the classroom. The web server connects with the database server to inquiry about these data. When the data is received, this data is sent in JavaScript object notation (JSON) format to the NodeMCU including the maximum number allowed and the actual number that existed.

At this moment, NodeMCU will compare if the actual number is less than 20 students, then this condition is satisfied and the NodeMCU will check the other condition, otherwise, the door will not open. The other condition is the temperature of the incoming person must be below (37.5) celsius. mlx90614 IR temperature sensor has been employed to measure the actual temperature of the incoming person. If the 
temperature is below the limit, the main NodeMCU will send the order to the solenoid lock to open the door. At the same time, NodeMCU will send notifications to the webserver to increase the actual number existed in the classroom by one. Otherwise, if the temperature of the person is equal to or higher than (37.5) celsius, the door will not open and an alarm will run. In addition, a notification will send to the cloud page including the actual temperature of the person and the time when the person gets closer from the door to the API that is created previously. The next Figure 3 shows a flow chart of the logical sequence that this proposed system follows.

Regarding the second ultrasonic sensor, it is located in the internal part of the system and attached to the main NodeMCU. This ultrasonic sensor is responsible to inform the main NodeMCU to open the door for any person who wants to leave the classroom. When the door has been opened, the main NodeMCU sends a notification as an action out to the webserver which in turn updates the SQL database by decreasing the actual number of students, existed in the classroom, by one. Generally, the main NodeMCU is responsible for checking the conditions, comparison processing, and communication with the webserver using HTTP requests. While the webserver is responsible for determining the maximum number of students allowed to enter, and updating the number of existed students. In this design system, the cloud is working locally but it can work publically over the internet, but it needs to be hosted by a public server.

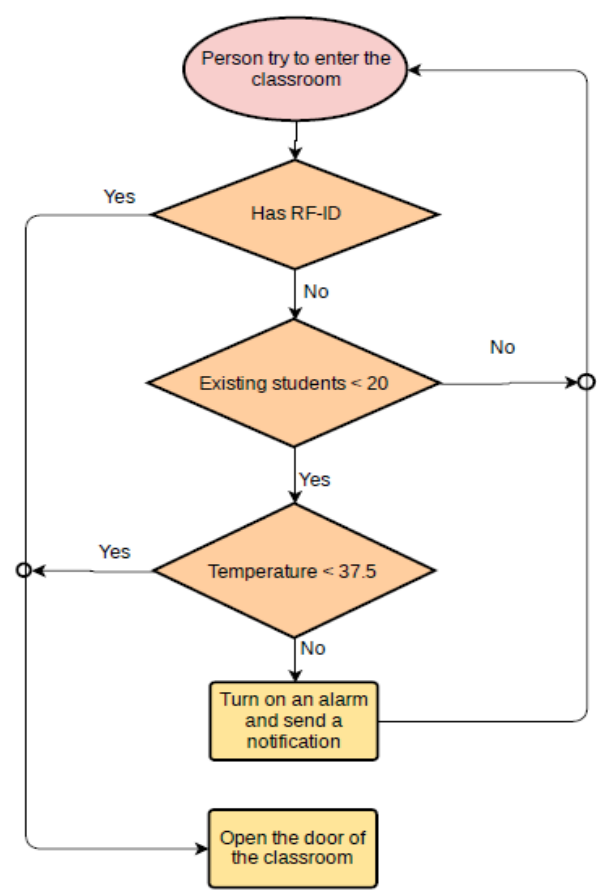

Figure 3. The flow chart of the logical sequence that this proposed system follows

\section{RESULTS AND DISCUSSION}

The structure of this design consists of an internal part and an external part. The designed system registers the students entering the classroom, when a student gets closer to the external part, this part will check the conditions. If the temperature of the student is lesser than the reference temperature and the number of students inside is lesser than the total allowed number, the lock will open the door for the student. Otherwise, if the temperature of the student is above the limit, the design system will run an alarm by sending notifications that appear on the cloud page including the real temperature of the student and the time at which he reached the door. The other scenario is when a student reaches the door and the classroom is full with the maximum number of students, then the door will not open until one student get out from the classroom or the authorities open the door using their radio frequency (RF) cards.

The internal part in turn is responsible to open the door for students who need to leave with the assistance of an Ultrasonic sensor. The instructor and authorities can get to the interface of the design system by utilizing the cloud page through the computer, iPad, and cell phone. The test is conducted and the design system is working perfectly. The next Figure 4 presents the interface of the cloud. It includes the number of persons inside the classroom, the maximum of people allowed to be inside, and the notification center. 


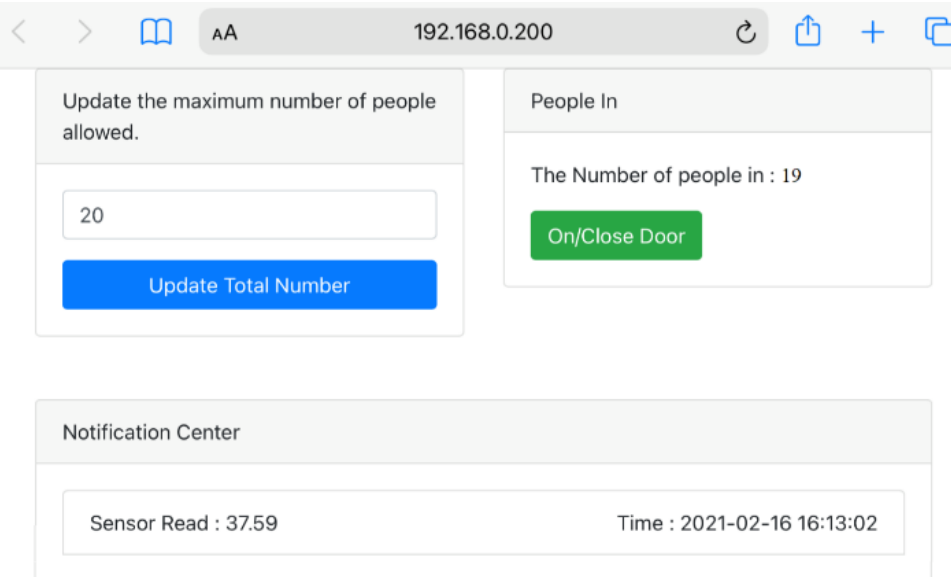

Figure 4. The interface of the cloud of the proposed system

\section{CONCLUSION}

This paper presents a planning framework to battle COVID-19 by utilizing cloud computing and a structure including Arduino (NodeMCU) with few sensors. In addition, collecting information about suspected individuals is a major perspective to save lives, and decrease COVID-19 effects on the economic and educational sectors. The activity parts of this planning framework, utilizing cloud computing that is included to decrease COVID-19 dangers, have been examined. This planning framework has an incredible impact on COVID19. This impact is relying upon keeping up the social distance with compelling checking for suspected cases. This planning framework can be utilized in various public territories, for example, air terminals, shopping centers, and schools. The features of cloud computing are used with the capability of NodeMCU to combat COVID-19. The work technique has been examined including a web server, my SQL database, and Arduino. The cloud interface has been accessible through a PC, iPad, and mobile phones, thus there will be no fear about the spread of COVID-19 through physical contact. This paper presents a robustness design that is proactive through using advanced algorithms to deal with the surrounding environment. This framework is important, reliable, and stable since the entire interaction is contactless.

\section{REFERENCES}

[1] H. M. Haglan, A. S. Mahmoud, M. H. Al-Jumaili, and A. J. Aljaaf, "New ideas and framework for combating COVID-19 pandemic using IoT technologies," Indonesian Journal of Electrical Engineering and Computer Science, vol. 22, no. 3, pp. 1565-1572, 2021, doi: 10.11591/ijeecs.v22.i3.pp1565-1572.

[2] N. A. Hidayat, P. Megantoro, A. Yurianta, A. Sofiah, S. A. Aldhama, and Y. A. Effendi, "The application of instrumentation system on a CONTACTLESS Robotic Triage assistant to detect early transmission on A covid-19 suspect," Indonesian Journal of Electrical Engineering and Computer Science, vol. 22, no. 3, pp. 1334-1344, 2021, doi: 10.11591/ijeecs.v22.i3.pp1334-1344.

[3] B. B. Gupta, D. P. Agrawal, S. Yamaguchi, and M. Sheng, "Soft computing techniques for big data and cloud computing," Soft Computing, vol. 24, no. 8, pp. 5483-5484, 2020, doi: 10.1007/s00500-020-04766-2.

[4] S. Logesswari, S. Jayanthi, D. KalaiSelvi, S. Muthusundari, and V. Aswin, "A study on cloud computing challenges and its mitigations," Materials Today: Proceedings, 2020, doi: 10.1016/j.matpr.2020.10.655.

[5] F. Fowley, C. Pahl, P. Jamshidi, D. Fang, and X. Liu, "A Classification and Comparison Framework for Cloud Service Brokerage Architectures," in IEEE Transactions on Cloud Computing, vol. 6, no. 2, pp. 358-371, 1 AprilJune 2018, doi: 10.1109/TCC.2016.2537333.

[6] A. M. R. Gali and V. R. Koduganti, "Dynamic and scalable virtual machine placement algorithm for mitigating side channel attacks in cloud computing," Materials Today: Proceedings, 2021, doi: 10.1016/j.matpr.2020.12.1136.

[7] M. Masdari and M. Zangakani, "Green Cloud Computing Using Proactive Virtual Machine Placement: Challenges and Issues," Journal of Grid Computing, vol. 18, no. 4, pp. 727-759, 2019, doi: 10.1007/s10723-019-09489-9.

[8] S. A. Butt, M. I. Tariq, T. Jamal, A. Ali, J. L. Díaz Martinez, and E. De-La-Hoz-Franco, "Predictive Variables for Agile Development Merging Cloud Computing Services," in IEEE Access, vol. 7, pp. 99273-99282, 2019, doi: 10.1109/ACCESS.2019.2929169.

[9] Z. Zhu, J. Peng, K. Liu, and X. Zhang, "A game-based resource pricing and allocation mechanism for profit maximization in cloud computing," Soft Computing, vol. 24, no. 6, pp. 4191-4203, 2019, doi: 10.1007/s00500-01904183-0.

[10] D. Tiwari and G. R. Gangadharan, "SecAuth-SaaS: a hierarchical certificateless aggregate signature for secure collaborative SaaS authentication in cloud computing," Journal of Ambient Intelligence and Humanized Computing, vol 12, pp. 10539-10563, 2021, doi: 10.1007/s12652-020-02864-5. 
[11] D. Gesvindr, O. Gasior, and B. Buhnova, "Architecture design evaluation of PaaS cloud applications using generated prototypes: PaaSArch Cloud Prototyper tool," Journal of Systems and Software, vol. 169, p. 110701, 2020, doi: $10.1016 /$ j.jss.2020.110701.

[12] C. Mouradian et al., "An IoT Platform-as-a-Service for NFV-Based Hybrid Cloud/Fog Systems," in IEEE Internet of Things Journal, vol. 7, no. 7, pp. 6102-6115, July 2020, doi: 10.1109/JIOT.2020.2968235.

[13] M. S. Sanaj and P. M. Joe Prathap, "Nature inspired chaotic squirrel search algorithm (CSSA) for multi objective task scheduling in an IAAS cloud computing atmosphere," Engineering Science and Technology, an International Journal, vol. 23, no. 4, pp. 891-902, 2020, doi: 10.1016/j.jestch.2019.11.002.

[14] K. Metwally, A. Jarray and A. Karmouch, "A Distributed Auction-based Framework for Scalable IaaS Provisioning in Geo-Data Centers," in IEEE Transactions on Cloud Computing, vol. 8, no. 3, pp. 647-659, 1 July-Sept. 2020, doi: 10.1109/TCC.2018.2808531.

[15] J. Rui and S. Danpeng, "Architecture Design of the Internet of Things Based on Cloud Computing," 2015 Seventh International Conference on Measuring Technology and Mechatronics Automation, 2015, pp. 206-209, doi: 10.1109/ICMTMA.2015.57.

[16] H. Kim, J. Park, and H. Jung, “Automatic control system based on IOT data identification," Indonesian Journal of Electrical Engineering and Computer Science, vol. 19, no. 3, pp. 1525-1532, 2020, doi: 10.11591/ijeecs.v19.i3.pp1525-1532.

[17] D. A. Al-Khaffaf and M. G. Al-Hamiri, "Performance evaluation of campus network involving VLAN and broadband multimedia wireless networks using OPNET modeler," TELKOMNIKA (Telecommunication, Computing, Electronics and Control), vol. 19, no. 5, pp. 1490-1497, Oct. 2021, doi: 10.12928/telkomnika.v19i5.18531.

[18] M. -F. R. Lee and T. -W. Chien, "Artificial Intelligence and Internet of Things for Robotic Disaster Response," 2020 International Conference on Advanced Robotics and Intelligent Systems (ARIS), 2020, pp. 1-6, doi: 10.1109/ARIS50834.2020.9205794.

[19] B. M. Nema, Y. Makki Mohialden, N. Mahmood Hussien, and N. Ali Hussein, "COVID-19 knowledge-based system for diagnosis in Iraq using IoT environment," Indonesian Journal of Electrical Engineering and Computer Science, vol. 21, no. 1, pp. 328-337, 2021, doi: 10.11591/ijeecs.v21.i1.pp328-337.

[20] L. Saba et al., "Six artificial intelligence paradigms for tissue characterisation and classification of non-COVID-19 pneumonia against COVID-19 pneumonia in computed tomography lungs," International Journal of Computer Assisted Radiology and Surgery, vol. 16, no. 3, pp. 423-434, 2021, doi: 10.1007/s11548-021-02317-0.

[21] L. Rafea, A. Ahmed, and W. D. Abdullah, "Classification of A COVID-19 dataset by using LABELS created from clustering algorithms," Indonesian Journal of Electrical Engineering and Computer Science, vol. 21, no. 1, pp. 164-173, 2021, doi: 10.11591/ijeecs.v21.i1.pp164-173.

[22] F. Shi et al., "Review of Artificial Intelligence Techniques in Imaging Data Acquisition, Segmentation, and Diagnosis for COVID-19," in IEEE Reviews in Biomedical Engineering, vol. 14, pp. 4-15, 2021, doi: 10.1109/RBME.2020.2987975.

[23] J. M. Scavuzzo et al., "Modeling the temporal pattern of Dengue, Chicungunya and Zika vector using satellite data and neural networks," 2017 XVII Workshop on Information Processing and Control (RPIC), 2017, doi: 10.23919/RPIC.2017.8211646.

[24] J. M. Scavuzzo, F. C. Trucco, C. B. Tauro, A. German, M. Espinosa and M. Abril, "Modeling the temporal pattern of Dengue, Chicungunya and Zika vector using satellite data and neural networks," 2017 XVII Workshop on Information Processing and Control (RPIC), 2017, pp. 1-6, doi: 10.23919/RPIC.2017.8211646.

[25] A. Florea and V. Fleaca, "Implementing an embedded system to identify possible COVID-19 suspects using thermovision cameras," 2020 24th International Conference on System Theory, Control and Computing (ICSTCC), 2020, pp. 322-327, doi: 10.1109/ICSTCC50638.2020.9259699.

[26] M. I. P. Nasution, N. Nurbaiti, N. Nurlaila, T. I. F. Rahma, and K. Kamilah, "Face Recognition Login Authentication for Digital Payment Solution at COVID-19 Pandemic," 2020 3rd International Conference on Computer and Informatics Engineering (IC2IE), 2020, pp. 48-51, doi: 10.1109/IC2IE50715.2020.9274654.

[27] S. Ahir, D. Telavane, and R. Thomas, "The impact of Artificial Intelligence, Blockchain, Big Data and evolving technologies in Coronavirus Disease - 2019 (COVID-19) curtailment," 2020 International Conference on Smart Electronics and Communication (ICOSEC), 2020, pp. 113-120, doi: 10.1109/ICOSEC49089.2020.9215294.

[28] A. W. Reza, M. M. Hasan, N. Nowrin, and M. M. Ahmed Shibly, "Pre-trained deep learning models in automatic COVID-19 DIAGNOSIS," Indonesian Journal of Electrical Engineering and Computer Science, vol. 22, no. 3, pp. 1540-1547, 2021, doi: 10.11591/ijeecs.v22.i3.pp1540-1547.

[29] A. I. Kyritsis and M. Deriaz, "A Queue Management Approach for Social Distancing and Contact Tracing," 2020 Third International Conference on Artificial Intelligence for Industries (AI4I), 2020, pp. 66-68, doi: 10.1109/AI4I49448.2020.00022.

[30] A. Ramchandani, C. Fan, and A. Mostafavi, "DeepCOVIDNet: An Interpretable Deep Learning Model for Predictive Surveillance of COVID-19 Using Heterogeneous Features and Their Interactions," in IEEE Access, vol. 8, pp. 159915-159930, 2020, doi: 10.1109/ACCESS.2020.3019989.

[31] C. T. Nguyen et al., "A Comprehensive Survey of Enabling and Emerging Technologies for Social DistancingPart I: Fundamentals and Enabling Technologies," in IEEE Access, vol. 8, pp. 153479-153507, 2020, doi: 10.1109/ACCESS.2020.3018140.

[32] S. Mandal and D. A. Khan, "A Study of Security Threats in Cloud: Passive Impact of COVID-19 Pandemic," 2020 International Conference on Smart Electronics and Communication (ICOSEC), 2020, pp. 837-842, doi: 10.1109/ICOSEC49089.2020.9215374. 